\title{
Effects analysis of imperfections on multi-antenna transceiving systems
}

\author{
Huiyong Li, Qi An*, Kexin Jia, Hao Wu and Zishu He
}

\begin{abstract}
Multi-antenna transceiving systems are widely used in high speed wireless communications due to its higher efficiency comparing to its traditional single-antenna counterparts. In this article, the performance effects of some inevitable imperfections are elaborated on multi-antenna transceiving systems. The simulation results are also displayed. An effective method for phase noise elimination is presented and validated using simulation examples.
\end{abstract}

\section{Multi-antenna technology scheme}

With the growing development of wireless communications in the current society, the conflict between the ever-increasing service requirements of wireless communications and the resource limitation of radio spectrum becomes increasingly apparent $[1,2]$. A key technology to solve this problem is multi-antenna techniques, which can enhance the frequency efficiency and link reliability drastically. Compared to the single-antenna communication systems, array gain (i.e., beam forming gain), diversity gain, multiplexing gain, and interference suppression can be obtained by using the multi-antenna communication systems.

In recent communication systems, two of the primary multi-antenna techniques are multi-input multi-output (MIMO) and transceiving beamforming.

MIMO [3,4], which is developed based on the smart antenna technology, has its distinct superiority. In the MIMO systems, the wireless channels are independent, or weakly correlated. Due to its abundant multi-paths, MIMO systems could enhance the system capability greatly [5].

The signal processing flow on transceiving beamforming is simple and apt to hardware applications because it does not involve so much prior knowledge and any complicated algorithm. However, the channels' consistency is so rigorous in demand. Thanks to the narrow beam, the performance will be decrease sharply if any direction error exists.

\footnotetext{
* Correspondence: sunnycaroline@163.com

School of Electronic Engineering, University of Electronic Science and Technology of China, Chengdu, China
}

(c) 2012 Li et al; licensee Springer. This is an Open Access article distributed under the terms of the Creative Commons Attribution License (http://creativecommons.org/licenses/by/2.0), which permits unrestricted use, distribution, and reproduction in any medium, provided the original work is properly cited.
According to these disadvantages, some inevitable imperfections will be discussed from four different aspects. In the following section, lots of closed-form expressions are derived and verified by the simulation results subsequently.

\section{Effects analysis of imperfect factors}

Considering an $N \times N$ transceiving beam formation system, there are also lots of imperfect elements even if the beams between transmitting and receiving ends are completely in the right direction. Some of these unsatisfactory factors such as narrowband or wideband amplitude and phase error, the amplitude and phase error in in-phase (I) channel, quadrature $(\mathrm{Q})$ channel, and phase noise, are focused in this article. Each of them is added into the ideal model and discussed, respectively (Figure 1).

\subsection{Narrowband amplitude and phase error}

In this study, we consider the case where the amplitude and phase error in the signal path are narrowband. Denote the output signal of filter in each transmitting path by $s(t)$, and the output of the $m_{t h}(m=1, \ldots, N)$ transmitting channel with error by $g_{m} e^{j \phi_{m}}$. Then the output of every transmitting antenna can be written as $g_{m} e^{j \phi_{m}} \cdot s(t)$, where $g_{m}=1-\sigma_{m}, \sigma_{m}$ is random and exponentially distributed, and $\varphi_{m}$ is uniformly distributed in the range of $[0, \theta]$. The $n_{t h}(n=1, \ldots, N)$ channel noise is denoted by $n_{n}(t)$. If the amplitude and phase error exists in each signal path at both the transmitting and receiving ends, which are expressed as $g_{m} e^{j \phi_{m}}$ and $h_{n} e^{j \varphi_{n}}$ respectively, the total signal received will be 


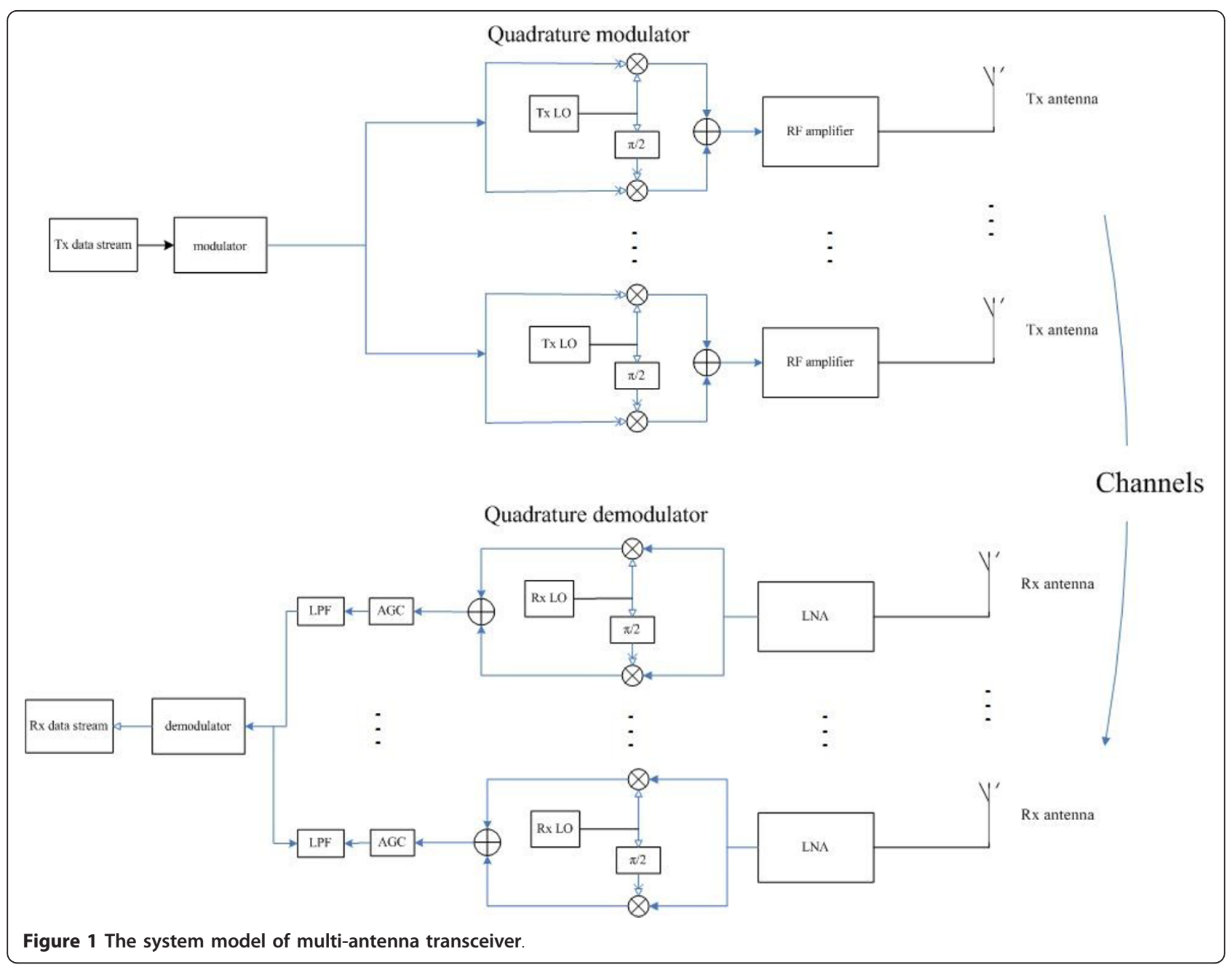

$$
\sum_{n=1}^{N}\left[h_{n} e^{j \varphi_{n}} \cdot\left(\left(\sum_{m=1}^{N} g_{m} e^{j \phi_{m}} \cdot s(t)\right)+n_{n}(t)\right)\right]
$$

In the multi-antenna transceiving systems, the counterparts of signal-to-noise ratio in single-antenna systems (SNR) will be updated to

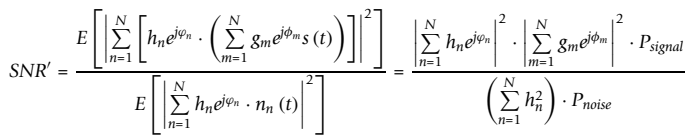

$$
\begin{aligned}
& =\frac{\left.\left|\sum_{n=1}^{N} h_{n} e^{\phi_{n}}\right|\right|_{n} ^{2} \cdot\left|\sum_{m=1}^{N} g m_{m} \dot{\phi}_{m}\right|^{2}}{\left(\sum_{n=1}^{N} h_{n}^{2}\right)} \cdot S N R
\end{aligned}
$$

that is,

$$
\begin{aligned}
& S N R^{\prime}(\mathrm{dB})=10 \log _{10}\left(\left|\sum_{n=1}^{N} h_{n} e^{i \varphi_{n}}\right|^{2}\right)+10 \log _{10}\left(\left|\sum_{m=1}^{N} g_{m} e^{i \phi_{m}}\right|^{2}\right) \\
& -10 \log _{10}\left(\sum_{n=1}^{N} h_{n}^{2}\right)+S N R(\mathrm{~dB})
\end{aligned}
$$

\subsection{Wideband amplitude and phase error}

When wideband amplitude and phase error is considered, the signal propogation channel is considered as a matrix [6]:

$$
\mathbf{h}=\left[\begin{array}{ccc}
h_{11} & \cdots & h_{1 N} \\
\vdots & \ddots & \vdots \\
h_{N 1} & \cdots & h_{N N}
\end{array}\right]
$$

where $h_{m n}$ represents the amplitude fading and phase difference between the $m_{t h}$ transmitting antenna and the $n$th receiving antenna (Figure 2).

After considering the multi-path transmission, $h_{m n}$ varies with time and can be modeled as [7]

$$
h_{m n}(t)=\delta(t) e^{j k d_{m n}}+b \delta(t-\tau) e^{j \phi_{m n}}
$$

where $\mathrm{b}$ is the relative amplitude fading, $\tau$ is the counter-shifting, $\varphi$ is the relative phase, $k=\frac{2 \pi}{\lambda}$ is the wave 


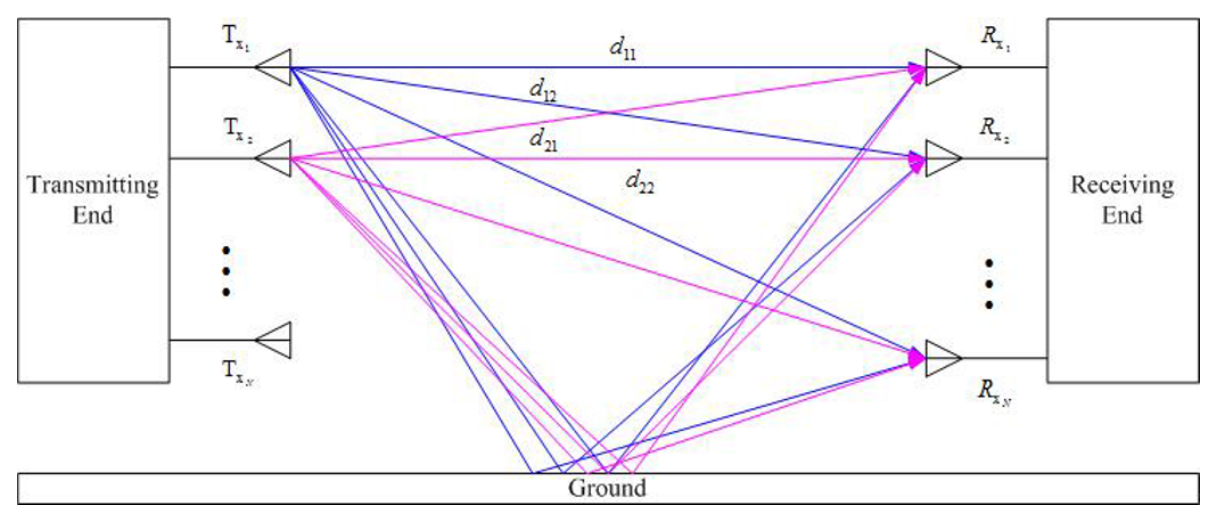

Figure 2 The setup of the Tx and Rx antennas

number and $d_{m n}$ is the distance between the $m$ th transmitting antenna and the $n$th receiving end. The Fourier transform of the channel matrix is

$$
H(\omega)=\left[\begin{array}{ccc}
e^{j k d_{11}} & \cdots & e^{j k d_{1 N}} \\
\vdots & \ddots & \vdots \\
e^{j k d_{N 1}} & \cdots & e^{j k d_{N N}}
\end{array}\right]+b e^{-j \omega \tau}\left[\begin{array}{ccc}
e^{j \phi d_{11}} & \cdots & e^{j \phi d_{1 N}} \\
\vdots & \ddots & \vdots \\
e^{j \phi d_{N 1}} & \cdots & e^{j \phi d_{N N}}
\end{array}\right]
$$

Thus, the channel's total power can be computed as

$$
P=\sum_{m=1}^{N} \sum_{n=1}^{N}\left|e^{j k d_{m n}}+b e^{-j \omega \tau} e^{j d_{m n}}\right|^{2}
$$

The channel gain is $G=10 \cdot \log _{10} P$.

The received signal vector can be expressed as

$$
\left[\begin{array}{c}
r_{1} \\
r_{2} \\
\vdots \\
r_{N}
\end{array}\right]=H \cdot\left[\begin{array}{c}
s_{1} \\
s_{2} \\
\vdots \\
s_{N}
\end{array}\right]+\left[\begin{array}{c}
n_{1} \\
n_{2} \\
\vdots \\
n_{N}
\end{array}\right]
$$

\subsection{Amplitude and phase error in in-phase and quadrature channels}

The procedure of extracting the in-phase and quadrature parts and up-converting brings amplitude and phase error to the receiving signal. The errors in the transmitting and receiving ends have similar expressions, which are modeled [8] as: $T_{m}(t)=2\left[k_{m} \cos (\omega t), \sin (\omega t\right.$ $\left.\left.+\phi_{m}\right)\right]$ and $R_{n}(t)=\left[l_{n} \cos (\omega t), \sin \left(\omega t+\gamma_{n}\right)\right]$. The $k_{m}$ and $l_{n}(m, n=1, \ldots, N)$ represent the amplitude errors and they are both in the range of $[1-\xi, 1+\xi]$, where $\xi$ is a tiny number. The phase errors $\phi$ and $\gamma$ are assumed to be much less than $\frac{\pi}{2}$. After the low-pass filtering, the received signal of the $n$th path is

$$
\begin{aligned}
r_{n}(t) & =\operatorname{LPF}\left\langle R_{n}(t) \cdot\left[\left(\sum_{m=1}^{N} T_{m}(t) \cdot s(t)\right)+n_{n}(t)\right]\right\rangle \\
& =\operatorname{LPF}\left\langle R_{n}(t) \cdot\left(\sum_{m=1}^{N} T_{m}(t)\right) \cdot s(t)+R_{n}(t) \cdot n_{n}(t)\right\rangle \\
& \triangleq H_{n} \cdot s(t)+n_{m}(t)
\end{aligned}
$$

where $H_{n}$ represents the $n$th channel matrix of I/Q channel and is defined as

$$
\begin{aligned}
& H_{n}=\operatorname{LPF}\left\langle R_{n}(t) \cdot\left(\sum_{m=1}^{N} T_{m}(t)\right)\right\rangle \\
& =\operatorname{LPF}\left\langle 2\left[l_{n} \cos (\omega t), \sin \left(\omega t+\gamma_{n}\right)\right]^{T} \cdot\left[\left(\sum_{m=1}^{N} k_{m}\right) \cos (\omega t), \sum_{m=1}^{N} \sin \left(\omega t+\varphi_{m}\right)\right]\right\rangle \\
& =\left[\begin{array}{cc}
l_{n} \cdot\left(\sum_{m=1}^{N} k_{m}\right) & l_{n} \cdot\left(\sum_{m=1}^{N} \sin \left(\varphi_{m}\right)\right) \\
\left(\sum_{m=1}^{N} k_{m}\right) \cdot \sin \left(\gamma_{n}\right) & \sum_{m=1}^{N} \cos \left(\gamma_{n}-\varphi_{m}\right)
\end{array}\right]
\end{aligned}
$$

The noise form in (8) is $\mathrm{n}_{n r}(t)=L P F\left\langle\mathrm{R}_{n}(t) n_{n}(t)\right\rangle$.

The sum of the signal from all $N$ channels is

$$
r(t)=\sum_{n=1}^{N} r_{n}(t)=\sum_{n=1}^{N}\left[H_{n} \cdot s(t)+n_{n r}(t)\right]
$$

\subsection{The model and correction of phase noise}

For both the single-antenna and multi-antenna systems, local oscillator (LO) will bring phase noise inevitably. Usually, the phase noise is assumed to obey the white Gaussian noise model, colored Gaussian noise model, or Wiener model [7,9-11]. Here we consider the Wiener model and denotes the phase noise for the $k$ th symbol by $e^{j \phi k}$. The discrete form of phase noise can be written as

$$
\varphi(k)=\alpha \cdot \varphi(k-1)+\Delta \varphi
$$




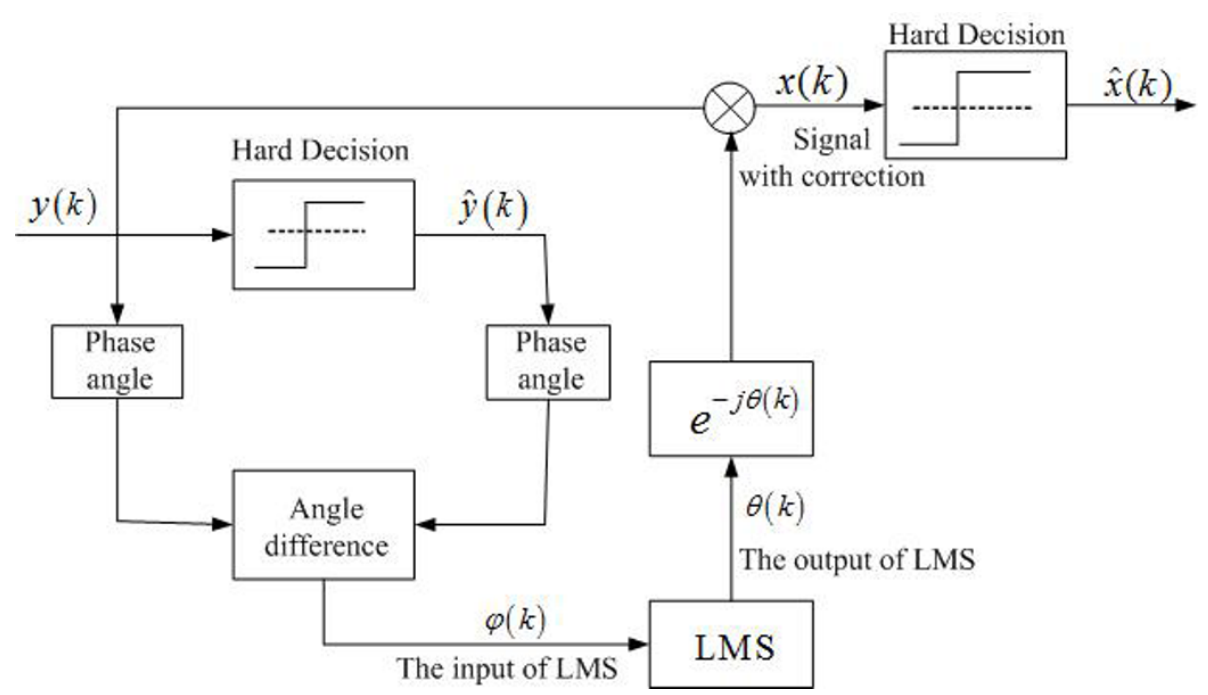

Figure 3 Algorithm structure of AO-LMS

where $\Delta \phi$ obeys the normal distribution and is random generated, that is, $\Delta \phi \sim N\left(0, \sigma^{2}\right), \sigma^{2}=\frac{4 \pi f_{\Delta 3 \mathrm{~dB}}}{F_{S}}$, $f_{\triangle 3 \mathrm{~dB}}$ is the $3 \mathrm{~dB}$ line width, $F_{s}$ is the sampling frequency, $\alpha$ is a constant near but less than 1 . The relational expression displayed in (9) indicates that the current phase noise is related with its previous moment, which is different to the two other counterparts. Assumed that $s(t)$ is the output signal in each transmitting path and all the transmitted signals can be received by every receiving antenna. The sampled received signal in the $n$th path can be written as

$$
r_{n}(k)=\left[N \cdot s(k)+n_{n}(k)\right] e^{j \varphi_{n}(k)}
$$

where $N$ denotes the number of antennas which is the same for both the transmitting and receiving ends. The sum of received signals is equivalent to $N^{2}$ times the transmitting signal in the ideal case. Then the error can be expressed as

$$
\begin{aligned}
e(k) & =\left(\frac{1}{N^{2}} \sum_{n=1}^{N} r_{n}(k)\right)-s(k) \\
& =\left(\frac{1}{N^{2}} \sum_{n=1}^{N}\left[\left(N \cdot s(k)+n_{n}(k)\right) \cdot e^{j \varphi_{n}(k)}\right]\right)-s(k) \\
& =s(k) \cdot\left[\left(\frac{1}{N} \sum_{n=1}^{N} e^{j \varphi_{n}(k)}\right)-1\right]+\frac{1}{N^{2}} \sum_{n=1}^{N} n_{n}(k) \cdot e^{j \varphi_{n}(k)}
\end{aligned}
$$

Thanks to the especial character, a few approaches are derived to eliminate the error $e(n)$. Gitlin [12] modeled the phase noise as sinusoidal signal and presented selfadaptation and compensation method named finite impulse response-adaptive line enhancer (FIR-ALE).
However, the ability of compensation is limited. Another method, the infinite impulse response-adaptive line enhancer (IIR-ALE) [13] has obvious effect provided many prior information being known. extended Kalman filter (EKF) [14] is used to estimate phase noise by linearizing first order digital phase locked loop (DPLL), in which, however, the non-convergent condition happens sometimes.

In this article a more accurate method is adopted which is called angle-offset least mean square (AOLMS). As shown in Figure 3, the angle difference between input signal and its hard-decision results is

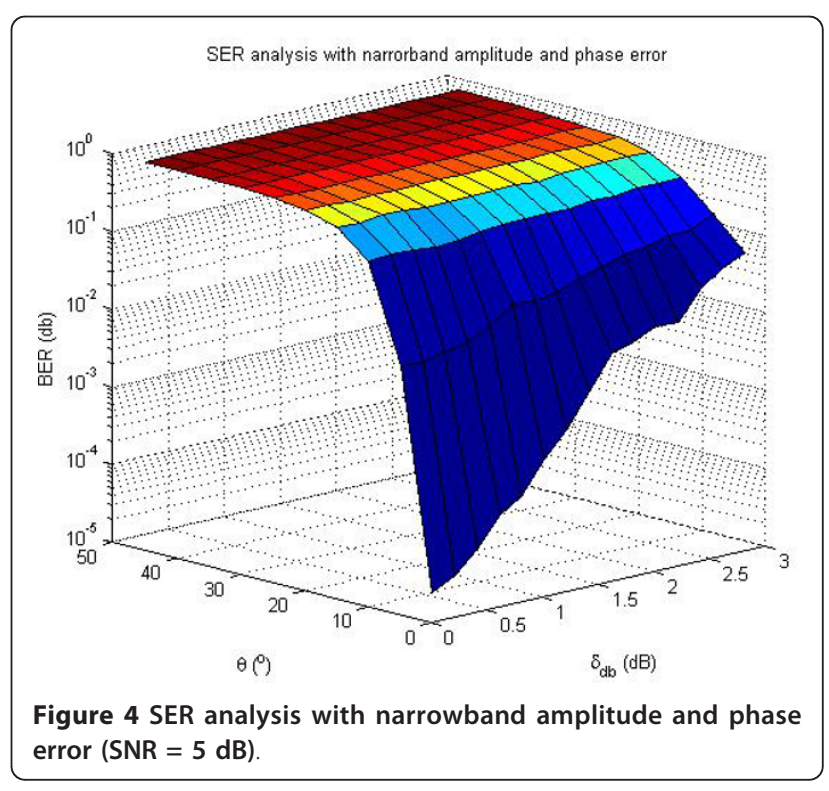




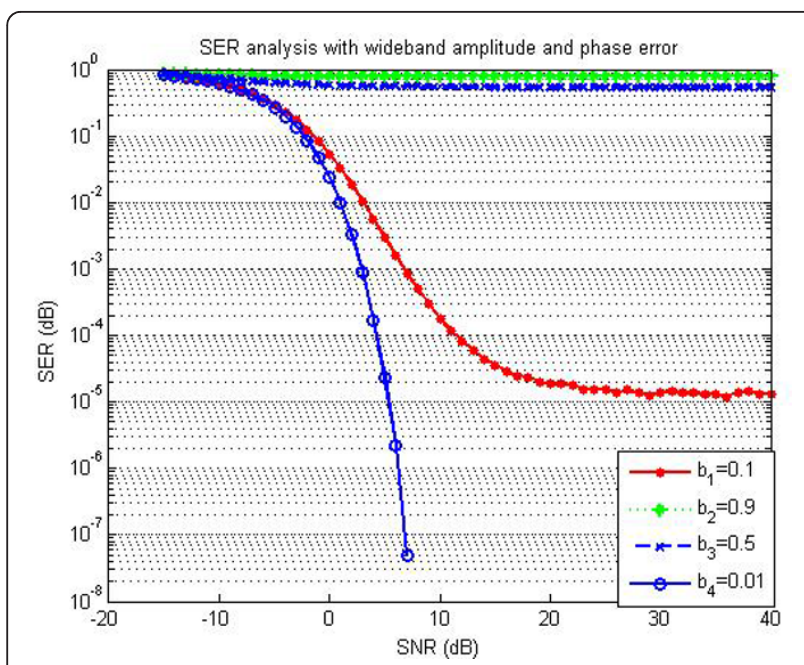

Figure 5 SER analysis with wideband amplitude and phase error.

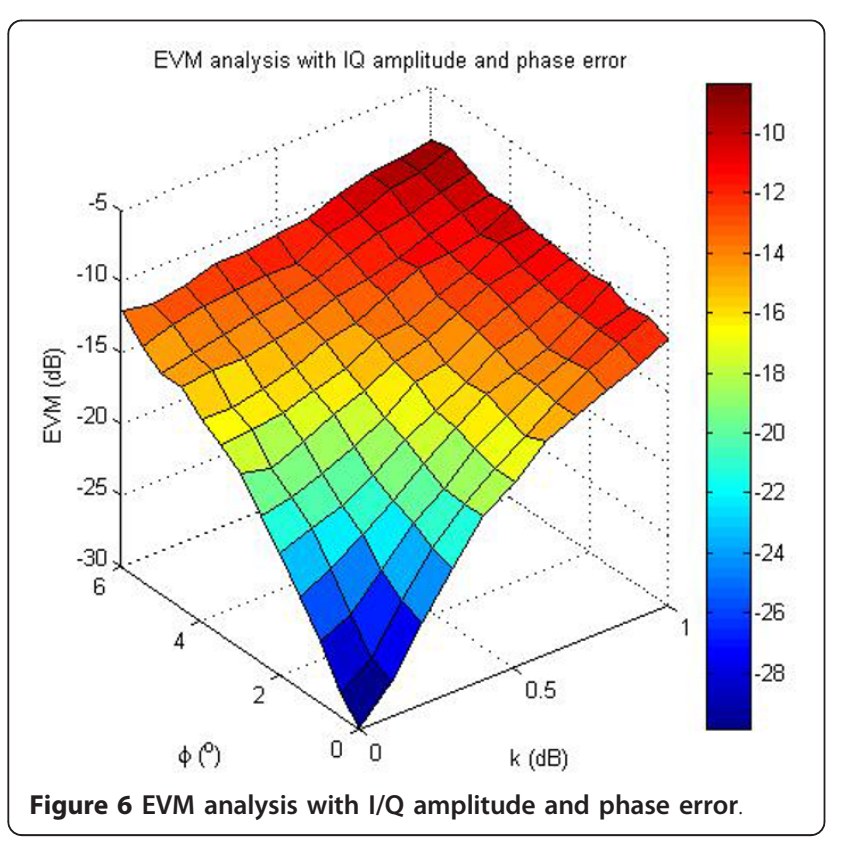

used as the input of LMS to estimate the real phase noise.

As an improvement approach to FIR-ALE, the input of LMS algorithm is a more accurate input, which is closer to the real phase offset caused by phase noise. It means a clearer aim and a better compensation ability.

\subsection{Simulation results}

The four errors introduced above are simulated. We consider a 64-ary QAM multi-antenna system. The transmitting and receiving ends each has 4 antennas. The sampling rate is $f_{s}=4 \mathrm{MHz}$. Rooted raised cosine (RRC) filters are adopted in both the transmitting and receiving ends. The roll-off factor is $\alpha=0.02$. The rate of sampling period and symbol period is 2 , and the order of RRC is 16. The effects of different factors on the transceiving performance are shown in Figures 4, 5, and 6. As is shown in Figure 4, symbol error rate (SER) gets a sharply increase when the narrowband phase error starts rising. Comparatively, the amplitude error puts a much less influence on it. Figure 5 displays the effect of wideband amplitude and phase error. When the channel fading is small (i.e., b1 $=0.01$ ), the situation is very similar to the ideal case. However, just a little increase of the fading can lead to the rapid rise of SER much less a large value near 1 . The amplitude and phase error caused in I/Q channels are measured by another criteria error vector magnitude (EVM) as it is shown in Figure 6. Yet the amplitude error and phase error both have a drastic influence on the transceiver's performance.

The following simulation results verify the compensation ability of AO-LMS. Constellation before and behind the block of phase noise compensation are shown in Figures 7 and 8 . In the view of constellation, the signal with phase noise brings a circular change along the amplitude circles. It is distinctly presented when SNR is large enough. Figure 8 shows that the algorithm adopted can take an apparent clustering even if large Gaussian channel noise exists. On the other hand, as is shown in
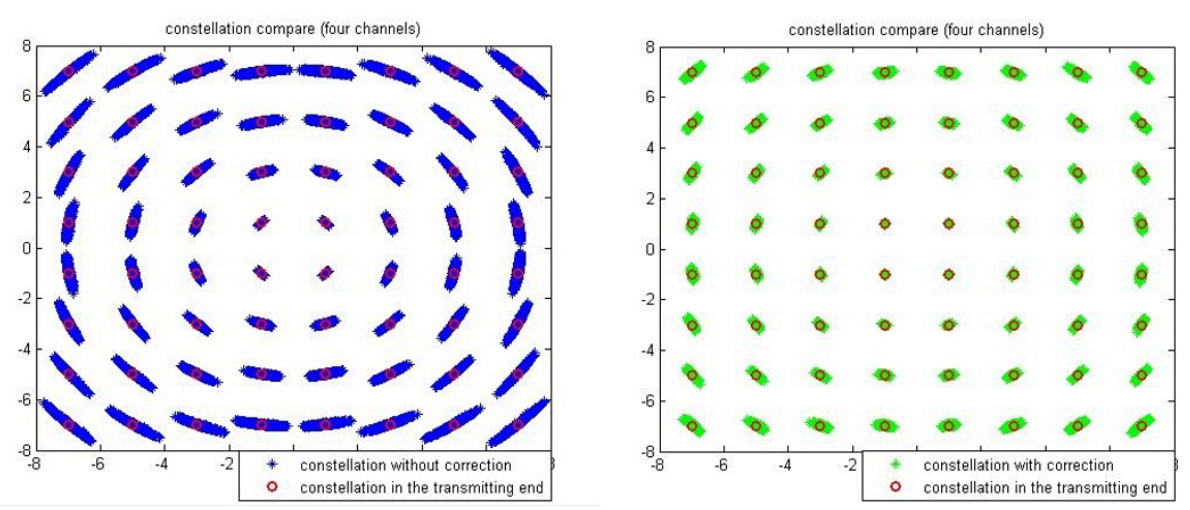

Figure 7 Constellations without and with phase noise compensation (SNR $=15 \mathrm{~dB})$. 

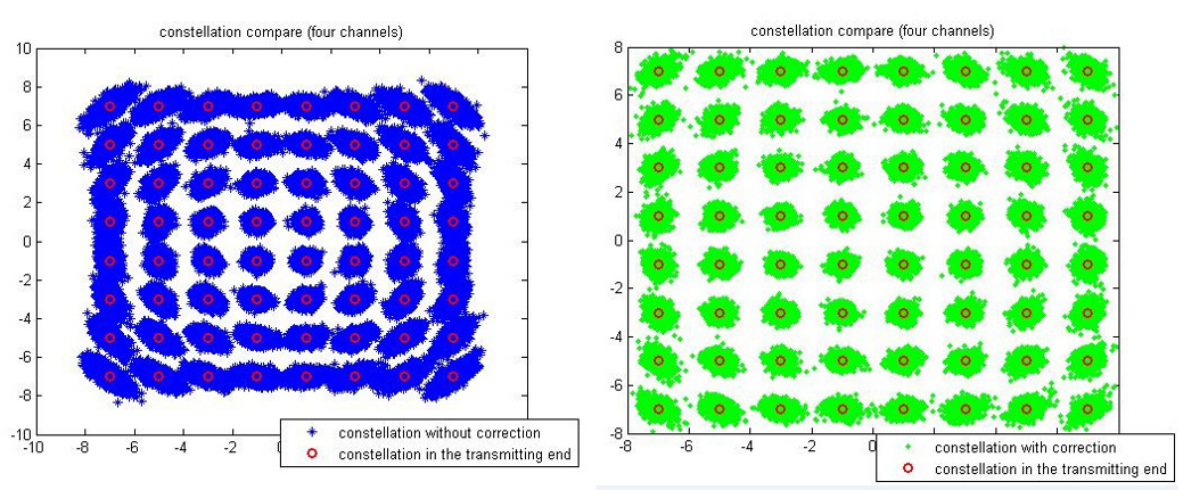

Figure 8 Constellations without and with phase noise compensation (SNR = $7 \mathrm{~dB}$ ).

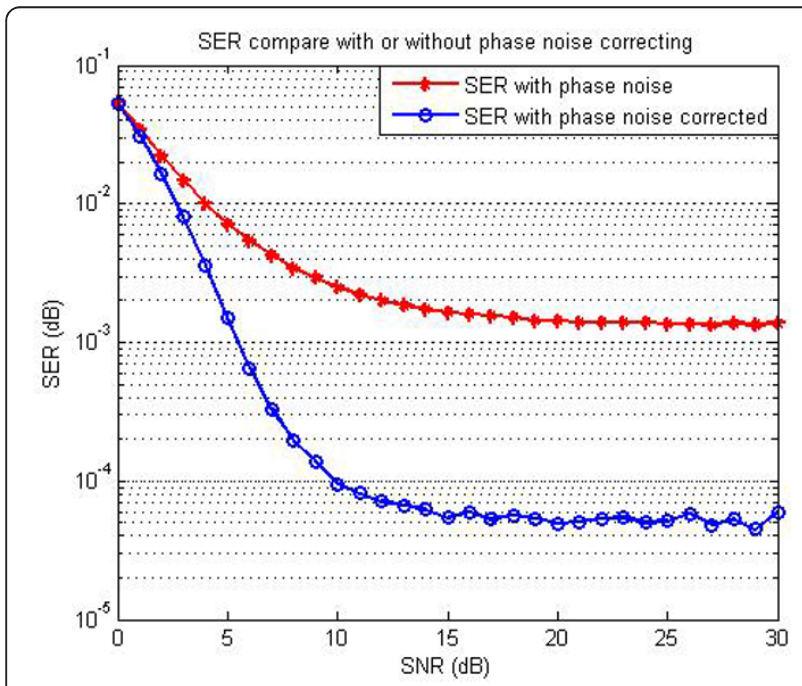

Figure 9 SER analysis before and behind the phase noise compensation.

Figure 9, the symbol error number can decrease sharply to less than one-tenth compared to the number before correction and at least one order of the SNR (dB) is gotten. Furthermore, the algorithm is adapted to engineering application due to its less tap number. The tap number used in the simulation is 16 .

\section{Conclusion}

Aiming at analyzing the transceiving beam forming systems, some imperfect factors are considered and analyzed theoretically. Phase noise is presented as a more inevitable factor. An effective compensation method is presented and verified by simulation results.

\section{Acknowledgements}

The authors would like to thank the support of Fundamental Research Funds for the Central Universities (ZYGX2010J015).

\section{Competing interests}

The authors declare that they have no competing interests.

Received: 28 September 2011 Accepted: 5 March 2012

Published: 5 March 2012

\section{References}

1. AJ Paulraj, RU Nabar, DA Gore, Introduction to space-time wireless communications [M], (Cambridge University Press, Cambridge, 2002)

2. X Liu, LT Yang, K Sohn, High-Speed Inter-view Frame Mode Decision Procedure for Multi-view Video Coding, Future Generation Computer Systems (Elsevier), May 2011, doi:10.1016/.future.2011.05.013

3. D Gesbert, M Shafi, From theory to practice: an overview of MIMO spacetime coded wireless systems. IEEE J Sel AreasCommun. 21(3), 281-302 (2003). doi:10.1109/JSAC.2003.809458

4. A Goldsmith, SA Jafar, N Jindal, S Vishwanath, Capacity limits of MIMO channels. IEEE J Sel Areas Commun. 21(5), 684-702 (2003). doi:10.1109/ JSAC.2003.810294

5. Q He, RS Blum, AM Haimovich, Non-coherent MIMO radar for location and velocity estimation: more antennas means better performance. IEEE Trans Signal Process. 58(7), 3661-3680 (2010)

6. T Ingason, H Liu, M Coldrey, A Wolfgang, J Hansryd, Impact of frequency selective channels on a line-of-sight MIMO microwave radio link. Vehicular Technology Conference (VTC 2010-Spring) 1-5 (2010)

7. A Georgiadis, Gain, phase imbalance, and phase noise effects on error vector magnitude. IEEE Trans Veh Technol. 53(2), 443-449 (2004). doi:10.1109/TVT.2004.823477

8. Z Chen, FF Dai, Effects of $L O$ phase and amplitude imbalances and phase noise on M-QAM transceiver performance. IEEE Trans Indust Electron. 57(5), 1505-1517 (2010)

9. NJeremy Kasdin, Discrete simulation of colored noise and stochastic processes and $1 / \mathrm{f}^{\mathrm{a}}$ power law noise generation. Proc IEEE. 83(5), 802-827 (1995). doi:10.1109/5.381848

10. S Berger, A Wittneben, Comparison of channel estimation protocols for coherent AF relaying networking in the presence of additive noise and LO phase noise. EURASIP J Wirel Commun Netw. 2010 (2010)

11. A Georgiadis, Gain, phase imbalance, and phase noise effects on error vector magnitude. IEEE Trans Veh Technol. 53(2), 443-449 (2004). doi:10.1109/TVT.2004.823477

12. $\mathrm{S}$ Wu, $\mathrm{P}$ Liu, Y Bar-Ness, Phase noise estimation and mitigation for OFDM systems. IEEE Trans Wirel Commun. 5(12), 3616-3625 (2006)

13. MR Gholami, S Nader-Esfahani, AA Eftekhar, A new method of phase noise compensation in OFDM. IEEE International Conference on Communications (ICC '03)3443-3446. Vol. 5, Issue Date 11-15 May 2003

14. B Farhang-Boroujeny, Pre-equaliser cancellation of sinusoidal phase jitter. IEE Proc Commun. 142(4), 216-220 (1995). doi:10.1049/ip-com:19952054

doi:10.1186/1687-1499-2012-81

Cite this article as: Li et al: Effects analysis of imperfections on multiantenna transceiving systems. EURASIP Journal on Wireless Communications and Networking 2012 2012:81. 